\title{
Exploring Multiple Dimensions of Young Women's Fertility Preferences in Malawi
}

\author{
Ashley Larsen Gibby \\ Brigham Young University - Provo, ashley_gibby@byu.edu \\ Nancy Luke \\ The Pennsylvania State University
}

Follow this and additional works at: https://scholarsarchive.byu.edu/facpub

Part of the Other Social and Behavioral Sciences Commons

\section{Original Publication Citation}

Gibby, Ashley Larsen and Nancy Luke. 2019. “Exploring Multiple Dimensions of Young Women's Fertility Preferences in Malawi." Maternal and Child Health Journal 23(11): 1508-1515.

\section{BYU ScholarsArchive Citation}

Gibby, Ashley Larsen and Luke, Nancy, "Exploring Multiple Dimensions of Young Women's Fertility Preferences in Malawi" (2019). Faculty Publications. 4794.

https://scholarsarchive.byu.edu/facpub/4794 


\title{
Exploring Multiple Dimensions of Young Women's Fertility Preferences in Malawi
}

\author{
Ashley Larsen Gibby ${ }^{1}$ (i) $\cdot$ Nancy Luke ${ }^{1}$
}

Published online: 21 June 2019

๑) Springer Science+Business Media, LLC, part of Springer Nature 2019

\begin{abstract}
Introduction Standard survey measures of fertility preferences, such as the desire for and preferred timing of future births, do not capture the complexity of individuals' preferences. New research focuses on additional dimensions of emotions and expectations surrounding childbearing. Few quantitative studies, however, consider the influence of all three dimensions of fertility preferences concurrently.

Methods Using longitudinal survey data from the Tsogolo la Thanzi project (2009-2012) in Malawi, this study employed logistic regression analysis to investigate the influence of young women's emotions, expectations, and a standard measure of fertility preferences on pregnancy and modern contraceptive use.

Results Young women experienced high unmet need; across survey waves, over three-quarters of women who desired a child in more than 2 years were not currently using modern contraceptives and over three-quarters of women who thought a pregnancy in the next month would be bad news (garnered from a measure of emotions surrounding pregnancy) were not currently using modern contraceptives. In regression models including all three measures of fertility preferences, each was significantly associated with the likelihood of a future pregnancy. The standard measure and emotions measure were significantly associated with modern contraceptive use.

Discussion Emotions and expectations surrounding pregnancy and childbirth appear to be distinct and salient aspects of fertility preferences in addition to the standard measure. A better understanding of the multidimensional nature of fertility preferences will help individuals define and achieve their reproductive goals and obtain appropriate services. Furthermore, future research should incorporate new measures of fertility preferences into surveys internationally.
\end{abstract}

Keywords Fertility intentions $\cdot$ Fertility preferences $\cdot$ Pregnancy $\cdot$ Contraceptives $\cdot$ Malawi

\section{Significance}

Fertility preferences are important predictors of reproductive outcomes, including births and contraceptive use, and are widely used to inform family planning programs. Surveys have historically measured fertility preferences as individuals' desires for children and their preferred timing of future births. Scholars now recognize that preferences are multidimensional and include additional factors, specifically emotions and expectations surrounding childbearing. To date, few studies have collected information on

Ashley Larsen Gibby

agl132@psu.edu

1 Department of Sociology and Criminology, The Pennsylvania State University, University Park, PA 16802-6027, USA multiple dimensions to enable assessments of their independent influences on reproductive outcomes. An examination that encompasses multiple dimensions and multiple outcomes will provide a more nuanced understanding of how fertility preferences influence women's reproductive decision-making.

\section{Background}

Since the 1960s, researchers have assessed individuals' fertility preferences through quantitative surveys with the goals of understanding reproductive decision-making and outcomes (e.g., Mumford et al. 2016). The earliest surveys, including the National Fertility Survey in the U.S. (Ryder and Westoff 1971) and family planning knowledge, attitudes, and practice (KAP) surveys in low-income countries 
(Casterline and Sinding 2000), included questions assessing respondents' future desire for a child and the preferred timing of that birth. These types of questions, which we refer to as "standard measures," are among the most commonly used indicators of fertility preferences. Research from across the globe has found that standard measures are associated with contraceptive use (e.g., Dodoo and Tempenis 2002; Testa 2012) and subsequent births (e.g., Machiyama et al. 2015; Testa et al. 2011; Testa and Rampazzo 2018). Consequently, standard measures are often used to inform family planning programs (Casterline and Sinding 2000).

The underlying assumption behind standard measures is that individuals purposefully map out whether and when to conceive their children (Aiken et al. 2016; Santelli et al. 2009). Many studies have found, however, inconsistencies between individuals' stated preferences and actual behavior. For example, the concept of unmet need arose from the finding that many sexually active, fecund women report that they do not want to get pregnant, yet they are not currently using contraceptives (Klerman 2000). Scholars have thus concluded that standard measures of fertility preferences cannot adequately capture the uncertainty, ambivalence, and complexity that surrounds childbearing (Aiken et al. 2016; Gomez et al. 2018).

In response to these critiques, subsequent research has focused on two additional dimensions of fertility preferences: emotions and expectations. Emotions refer to individuals' affective responses to pregnancy. This concept draws on the theory of planned behavior, which posits that attitudes toward a behavior, whether a favorable or unfavorable appraisal, can influence individuals' actions (Asare 2015). Emotions are often assessed retrospectively by asking respondents their degree of happiness in reaction to a past pregnancy (Santelli et al. 2009). Retrospective measures are vulnerable to ex-post rationalization (i.e., individuals report more positive emotions after a birth has occurred than they would have before the pregnancy), however, and could therefore be unreliable. Accordingly, recent studies have assessed emotions using prospective measures that gauge individuals' happiness about the possibility of becoming pregnant (Aiken 2015; Jones 2017; Speizer 2006).

Scholars have suggested that emotional reactions to pregnancy or childbirth could be as important as standard measures of fertility preferences in predicting reproductive outcomes (Aiken et al. 2016; Hartnett 2012; Jones 2017). Although few studies consider both dimensions concurrently, one U.S. study found that women's happiness regarding a previous pregnancy was negatively related to terminating the pregnancy after accounting for standard measures of fertility preferences (Santelli et al. 2009). An additional study found that happiness was positively related to women's contraceptive use net of standard measures (Geist et al. 2019).
While standard measures refer to abstract preferences, such as the desire for children or ideal timing of a birth, a third dimension concerns realistic expectations regarding future fertility. Assessment of expectations draws on individuals' ability to forecast their own realities, and survey measures have assessed individuals' expectations about becoming pregnant or certainty about future births, for example (Barrett and Wellings 2002; Stanford et al. 2000). One early study used data from the National Fertility Survey and found that women's expectations about the number of children they would have were highly correlated with a standard measure of future desire for a pregnancy (Ryder and Westoff 1971). Thereafter, some scholars used the concepts of fertility desires and expectations interchangeably (e.g., Hayford 2009; Morgan 2001). Several studies, however, support the view that expectations are a distinct determinant of reproductive outcomes. For example, one U.S. study found that women's positive expectations about the timing of the next birth were associated with a subsequent birth after accounting for standard measures of fertility preferences (Schoen et al. 1999).

In sum, although a large body of research points to the multidimensional nature of fertility preferences, many studies continue to rely on standard measures. A small number consider the influence of two or more dimensions concurrently. In this study, we use longitudinal survey data from young women in Malawi to estimate the independent influence of three dimensions of fertility preferences-a standard measure, emotions, and expectations-on subsequent pregnancy and modern contraceptive use.

\section{Methods}

\section{Data and Sample}

Malawi has recently begun a fertility transition with an attendant decrease in the total fertility rate (TFR), which had been among the highest in the world. The TFR was 5.7 births per woman in 2010, falling to 4.4 in 2015-2016 (NSO and ICF Macro 2017). Contraceptive prevalence rates have recently begun to climb, and $46.7 \%$ of women aged 20-24 used modern contraceptives (sterilization, injectables, intrauterine devices, contraceptive pills, implants, condoms, calendar method, lactational amenorrhea, and emergency contraceptives $^{1}$ ) in 2015-2016 (NSO and ICF Macro 2017).

\footnotetext{
1 The Malawi Demographic and Health Survey (DHS) also records use of traditional contraceptive methods including the rhythm method, withdrawal, and any other traditional methods reported by respondents (NSO and ICF Macro 2017).
} 
We used data from the Tsogolo la Thanzi project (TLT), a longitudinal survey project in Balaka District, Malawi, designed to examine the fluctuating nature of childbearing decisions of young adults. TLT followed a random sample of 1505 women ages 15-25 for 3 years (May 2009-June 2012). Respondents were interviewed every 4 months, creating eight waves of data. The study was approved by the IRBs of the Pennsylvania State University and the University of Chicago, and all participants gave informed consent prior to their inclusion. A detailed description of the sampling design and methods is available on the TLT website (https ://tsogololathanzi.uchicago.edu/).

The unit of analysis for our study was the person-wave. Each woman was interviewed in up to eight waves of the survey, and thus each wave for each woman was designated as a person-wave. We began by pooling all eight waves of data for all women, resulting in 10,876 person-waves. Our analytic sample was restricted to women in each wave who were at risk of becoming pregnant. Therefore, we excluded person-waves in which women were pregnant $(n=1326)$ and in which women reported having no sexual partners or romantic relationships $(n=1267)$. Finally, we excluded person-waves in which women were sterilized $(n=45)$. The final analytic sample included 8238 person-waves (1498 respondents).

\section{Measures}

All variables were constructed for each survey wave. The dependent variable pregnancy was coded dichotomously and lagged 4 months to ensure that fertility preferences at time one predicted pregnancy at time two. Pregnancy status was assessed through rapid urine tests. The dependent variable current modern contraceptive use indicated whether respondents reported using a modern method or not.

We constructed a standard measure of fertility preferences using responses to the question, "How long would you like to wait before having your first/next child?" We created a categorical variable indicating whether respondents wanted a child in less than 2 years, 2 years or more, or did not want a/another child. With respect to emotions, respondents were asked, "If you found out you were pregnant next month, would that news be: very bad, fairly bad, neither good nor bad, fairly good, very good, or don't know?" We created a trichotomous variable indicating good news, bad news, or neither good nor bad news. In $0.04 \%$ of personwaves $(n=4)$, women responded that they did not know whether it would be good news or bad news. These cases were coded as missing.

Respondents' expectations toward future childbearing were assessed through a unique interactive exercise (Trinitapoli and Yeatman 2011). Respondents were asked the likelihood of getting pregnant or having a child in the next year. The interviewer then placed 10 beans on the table and respondents were instructed to shift to a plate the number of beans that represented the likelihood of the event happening: 0 indicating that a pregnancy or birth would certainly not happen and 10 meaning that a pregnancy or birth was certain to happen. This measure ranged from 0 to 10 .

We adjusted for respondents' background characteristics, including age, education, marital status (married or not), number of children, if they had a birth in the previous two years or not, and survey wave (1-8). In addition, we accounted for economic status by constructing an index based on household assets and housing materials and standardizing it to mean zero and standard deviation one. ${ }^{2}$

\section{Analytic Strategy}

Analyses were conducted in Stata 14 with all logistic regression models adjusted for controls and clustered at the person-level to account for dependence between multiple observations of the same woman across person-waves. Four regression models were estimated with pregnancy as the dependent variable: one for each of the three measures of fertility preferences separately and one including all three measures simultaneously. The same approach was utilized for models with current modern contraceptive use as the dependent variable. To avoid list-wise deletion, missing data were handled through multiple imputation. ${ }^{3}$

\footnotetext{
${ }^{2}$ Respondents indicated whether or not their households owned items including a bed with a mattress, television, radio, landline/ mobile phone, refrigerator, and vehicle (coded owned the item $=1$, did not own $=0$ ); their main source of water (safer sources coded higher); and main material used for the floor of the house (more expensive materials coded higher). To create the index, a factor score was generated through principal components analysis utilizing the full sample of women across waves. The resulting asset scores were then standardized in relation to a standard normal distribution with a mean of zero and a standard deviation of one. We utilized an approach similar to the DHS (NSO and ICF Macro 2017; Rutstein and Johnson 2004).

3 The variables included here had varying degrees of missing data. Two variables had no missing values (birth in the last 2 years and survey wave). Other variables had less than $6 \%$ of values missing (standard measure of fertility preferences, emotions, expectations, age, education, marital status, number of children, and economic status). Information on pregnancy in the subsequent 4 months was missing in $27 \%$ of person-waves, as numerous women were not located and interviewed 4 months later and a pregnancy test was thus not completed. All missing data were imputed to avoid list-wise deletion (Rubin 2004). Imputation of new data sets (estimating new values each time) can be limitless; however, 20 iterations is an acceptable threshold (Johnson and Young 2011). We performed 25 iterations to ensure confidence in our results.
} 
Table 1 Characteristics of women at baseline, Balaka, Malawi $(n=1498)$

\begin{tabular}{llr}
\hline Categorical variables & Non-missing N & Percent \\
\hline Pregnancy during study period (2009-2012) & 1400 & 48.60 \\
Married & 1498 & 41.39 \\
Had a birth in previous 2 years & 1498 & 19.09 \\
\hline Continuous variables & Non-missing N & Mean \pm SD \\
\hline Age in years & 1498 & $19.53 \pm 3.29$ \\
Education in years & 1479 & $7.65 \pm 2.82$ \\
No. of children & 1496 & $0.80 \pm 0.96$ \\
Economic status (standardized index) & 1495 & $0.02 \pm 2.46$ \\
\hline
\end{tabular}

Source Tsogolo la Thanzi 2009-2012

Table 2 Characteristics of respondents across person-waves by measures of fertility preferences and by modern contraceptive use, Balaka, Malawi $(\mathrm{n}=8238)$

\begin{tabular}{|c|c|c|c|c|}
\hline \multirow[t]{2}{*}{ Categorical variables } & \multirow{2}{*}{ Non-missing $\mathrm{N}$} & \multirow{2}{*}{$\begin{array}{l}\text { Total sample } \\
\text { Percent }\end{array}$} & \multirow{2}{*}{$\begin{array}{l}\text { Currently using modern } \\
\text { contraceptives } \\
\text { Percent }\end{array}$} & \multirow{2}{*}{$\begin{array}{l}\text { Not currently using modern } \\
\text { contraceptives } \\
\text { Percent }\end{array}$} \\
\hline & & & & \\
\hline $\begin{array}{l}\text { Pregnant in the subsequent survey wave (4 months } \\
\text { later) }\end{array}$ & 5374 & 10.24 & 8.63 & 11.41 \\
\hline Current modern contraceptive use & 7955 & 42.39 & - & - \\
\hline \multicolumn{5}{|l|}{ Standard measure } \\
\hline \multicolumn{5}{|l|}{ Wants a child in } \\
\hline Less than 2 years & 7943 & 15.42 & 10.98 & 18.70 \\
\hline Two years or more & 7943 & 77.49 & 79.26 & 76.19 \\
\hline Doesn't want a child & 7943 & 7.08 & 9.76 & 5.11 \\
\hline \multicolumn{5}{|l|}{ Emotions } \\
\hline \multicolumn{5}{|l|}{ Thinks pregnancy next month would be } \\
\hline Bad news & 7821 & 81.22 & 83.28 & 79.66 \\
\hline Good news & 7821 & 15.88 & 12.88 & 18.14 \\
\hline Neither bad nor good news & 7821 & 2.90 & 3.84 & 2.20 \\
\hline Continuous variable & & Mean \pm SD & Mean \pm SD & Mean \pm SD \\
\hline \multicolumn{5}{|l|}{ Expectations } \\
\hline $\begin{array}{l}\text { Probability of pregnancy or birth in the next year } \\
\text { (mean number of beans) })^{\mathrm{a}}\end{array}$ & 7953 & 2.65 & $2.64 \pm 2.83$ & $2.65 \pm 3.11$ \\
\hline
\end{tabular}

Source Tsogolo la Thanzi 2009-2012

${ }^{a}$ On a scale $0-10$, where 0 beans indicates that a pregnancy or birth will certainly not occur; 10 beans indicates that a pregnancy or birth certainly will occur in the next year

\section{Results}

Table 1 presents an overview of respondents. Across all eight waves of the study period, $48.6 \%$ of women became pregnant. At baseline (the wave women entered the survey), $41 \%$ of women were married. The mean age of the sample was 19.5 years and women had 0.8 children on average.

Table 2 refers to characteristics of person-waves. In $10.2 \%$ of all the waves in which woman were interviewed, a pregnancy occurred by the subsequent survey wave (4 months later). Women were using modern contraceptives in $42.4 \%$ of person-waves. Across $77.5 \%$ of personwaves, women wanted to delay the next child for two or more years. Across person-waves, $81.2 \%$ of women felt that a pregnancy in the next month would be bad news and $15.9 \%$ thought it would be good news. Women estimated a $27 \%$ likelihood of a pregnancy or birth in the next year 
across person-waves ( 2.7 out of 10 beans were indicated on average). The correlation between the three fertility preferences measures was not high $(r=0.5$ for the standard measure and emotions, $r=0.4$ for the standard measure and expectations, and $r=0.4$ for emotions and expectations), providing an initial indication that they capture distinct aspects of fertility preferences.

Table 2 also presents tabulations of respondent characteristics among users and non-users of modern contraceptives. A pregnancy occurred 4 months later in $8.6 \%$ of personwaves in which women reported using modern contraceptives and in $11.4 \%$ of person-waves in which they did not use modern contraceptives. ${ }^{4}$

Across person-waves in which women were not using modern contraceptives, three quarters desired a child in two or more years (76.2\% of person-waves) or never $(5.1 \%)$ and three-quarters thought it would be bad news to become pregnant in the next month (79.7\%). These figures underscore the high unmet need apparent in this young population, as these women desired to postpone or cease childbearing or felt a pregnancy would be unwelcome but were not using modern contraceptives at the time.

Table 3 displays results from logistic regression models that examined the relationships between the three dimensions of fertility preferences and the likelihood of pregnancy in the next 4 months. Models 1-3 reveal that each dimension was significantly associated with subsequent pregnancy after adjusting for background characteristics. All three dimensions continued to be significant and in the expected directions when they were included in the same model (Model 4). For example, compared to women who wanted a pregnancy in less than 2 years (reference), the odds of subsequent pregnancy were significantly smaller for women who wanted a/another child in 2 years or more or did not want a/ another child. Women who thought a pregnancy would be bad news were significantly less likely to become pregnant than women who thought a pregnancy would be good news (reference). Finally, women who had greater expectations that they would experience pregnancy or birth in the next year were more likely to experience a pregnancy; a shift in one bean, which indicated a $10 \%$ increase in their expectations, was associated with a $7 \%$ increase in the likelihood of a pregnancy. Across all models, childbirth in the last

\footnotetext{
${ }^{4}$ Each woman was interviewed up to eight times in the survey across 3 years. Approximately half of the women in our sample became pregnant across the 3 years, however they were recorded as becoming pregnant (positive pregnancy test) in as few as one of the waves. Therefore, the percentage of women becoming pregnant is much higher than the percentage of survey waves (person-waves) where they were coded as becoming pregnant.
}

Table 3 Adjusted odds ratios from logistic regressions of women who became pregnant within 4 months, Balaka, Malawi $(n=8238$ person-waves)

Model 1 Model 2 Model 3 Model 4

\begin{tabular}{|c|c|c|c|c|}
\hline \multicolumn{5}{|l|}{ Standard measure } \\
\hline \multicolumn{5}{|l|}{ Wants a child in } \\
\hline Less than 2 years $(r)$ & \multicolumn{3}{|l|}{1.00} & 1.00 \\
\hline Two years or more & \multicolumn{3}{|l|}{$0.35 * * *$} & $0.62 * * *$ \\
\hline Doesn't want a child & \multicolumn{3}{|l|}{$0.17 * * *$} & $0.38 * * *$ \\
\hline \multicolumn{5}{|l|}{ Emotions } \\
\hline \multicolumn{5}{|c|}{ Thinks pregnancy next month would be } \\
\hline Good news (r) & & \multicolumn{2}{|l|}{1.00} & 1.00 \\
\hline Bad news & & \multicolumn{2}{|l|}{$0.30 * * *$} & $0.49 * * *$ \\
\hline $\begin{array}{l}\text { Neither good nor bad } \\
\text { news }\end{array}$ & & \multicolumn{2}{|l|}{$0.67 *$} & 0.91 \\
\hline \multicolumn{5}{|l|}{ Expectations } \\
\hline $\begin{array}{l}\text { Probability of pregnancy } \\
\text { or birth in the next year }\end{array}$ & & & $1.14 * * *$ & $1.07 * * *$ \\
\hline Age (years) & 1.01 & 1.01 & 1.03 & 0.99 \\
\hline Education (years) & 0.97 & $0.97^{\dagger}$ & $0.96^{*}$ & 0.98 \\
\hline Married & 1.15 & 1.04 & 1.14 & 0.98 \\
\hline Number of children & 1.00 & 0.93 & $0.87^{\dagger}$ & 1.03 \\
\hline Birth in last 2 years & $0.55 * * *$ & $0.57 * * *$ & $0.46 * * *$ & $0.64 * * *$ \\
\hline Economic status & $0.91 * * *$ & $0.91 * * *$ & $0.92 * * *$ & $0.92 * * *$ \\
\hline Survey wave (1-8) & $1.07 * *$ & $1.06 *$ & 1.04 & $1.05^{*}$ \\
\hline
\end{tabular}

Source Tsogolo la Thanzi 2009-2012

$r$ reference group

$* * * \mathrm{p}<.001 * * \mathrm{p}<.01 * \mathrm{p}<.05{ }^{\dagger} \mathrm{p}<.1$

${ }^{\mathrm{a}}$ On a scale $0-10$, where 0 beans indicates that a respondent expects a pregnancy or birth will certainly not occur; 10 beans indicates that a respondent expects a pregnancy or birth certainly will occur in the next year

2 years and economic status were negatively and significantly related to subsequent pregnancy.

Table 4 displays results from regression models for current modern contraceptive use. Models 1-3 reveal that each dimension was significantly associated with modern contraceptive use after adjusting for background characteristics. When all three dimensions were included in the same model (Model 4), the standard measure and emotions measure were significantly related to contraceptive use in the expected directions. Women who wanted to wait two or more years for a birth and women who thought a pregnancy would be bad news were significantly more likely to use modern contraceptives. Expectations were no longer significantly associated with modern contraceptive use, however.

Across all models in Table 4, education, marriage, number of children, having had a birth in the last 2 years, and survey wave were positively associated with modern contraceptive use. 
Table 4 Adjusted odds ratios from logistic regressions of women who were currently using modern contraceptives, Balaka, Malawi $(\mathrm{n}=8238$ person-waves $)$

Model 1 Model 2 Model 3 Model 4

\begin{tabular}{|c|c|c|c|c|}
\hline \multicolumn{5}{|l|}{ Standard measure } \\
\hline \multicolumn{5}{|l|}{ Wants a child in } \\
\hline Less than 2 years $(r)$ & \multicolumn{3}{|l|}{1.00} & 1.00 \\
\hline Two years or more & \multicolumn{3}{|l|}{$1.92 * * *$} & $1.63 * * *$ \\
\hline Doesn't want a child & \multicolumn{3}{|l|}{$1.59 * *$} & 1.32 \\
\hline \multicolumn{5}{|l|}{ Emotions } \\
\hline \multicolumn{5}{|c|}{ Thinks pregnancy next month would be } \\
\hline Good news (r) & & \multicolumn{2}{|l|}{1.00} & 1.00 \\
\hline Bad news & & \multicolumn{2}{|l|}{$1.74 * * *$} & $1.38 * *$ \\
\hline $\begin{array}{l}\text { Neither good nor bad } \\
\text { news }\end{array}$ & & \multicolumn{2}{|l|}{$1.62 * *$} & $1.35^{\dagger}$ \\
\hline \multicolumn{5}{|l|}{ Expectations } \\
\hline $\begin{array}{l}\text { Probability of pregnancy } \\
\text { or birth in the next year }\end{array}$ & & & $0.97 *$ & 1.00 \\
\hline Age (years) & 1.00 & 0.99 & 0.98 & 1.01 \\
\hline Education (years) & $1.12 * * *$ & $1.12 * * *$ & $1.13 * * *$ & $1.12 * * *$ \\
\hline Married & $2.09 * * *$ & $2.14 * * *$ & $2.02 * * *$ & $2.15^{* * *}$ \\
\hline Number of children & $1.61 * * *$ & $1.64 * * *$ & $1.70 * * *$ & $1.61 * * *$ \\
\hline Birth in last 2 years & $1.43 * * *$ & $1.45^{* * *}$ & $1.58 * * *$ & $1.40 * * *$ \\
\hline Economic status & 0.99 & 0.99 & 0.99 & 0.99 \\
\hline Survey wave (1-8) & $1.07 * * *$ & $1.07 * * *$ & $1.07 * * *$ & $1.07 * * *$ \\
\hline
\end{tabular}

Source Tsogolo la Thanzi 2009-2012

$r$ reference group

$* * * \mathrm{p}<.001 * * \mathrm{p}<.01 * \mathrm{p}<.05{ }^{\dagger} \mathrm{p}<.1$

${ }^{\mathrm{a}}$ On a scale $0-10$, where 0 beans indicates that a respondent expects a pregnancy or birth will certainly not occur; 10 beans indicates that a respondent expects a pregnancy or birth certainly will occur in the next year

\section{Discussion}

We explored three dimensions of young women's fertility preferences and links to reproductive behavior using longitudinal survey data from Malawi. An extensive literature argues that fertility preferences are not fully captured by standard measures, such as the desire for a child and the preferred timing of a birth (e.g., Aiken et al. 2016; Jones 2017). Subsequent research focuses on two additional dimensions - emotions and expectations surrounding childbearing (e.g., Geist et al. 2019; Santelli et al. 2009; Schoen et al. 1999) - yet this work rarely examines all of these components together. We took a more holistic approach and assessed the independent influence of all three dimensions on two reproductive outcomes: future pregnancy and current modern contraceptive use.

We used data from the Tsogolo la Thanzi project, which collected information on multiple dimensions of fertility preferences, a notable advantage over many studies internationally. In regression models including all three dimensions - the standard measure, emotions, and expectations - and controlling for women's background characteristics, we found that all three were significantly associated with a subsequent pregnancy. The standard measure and emotions measure were significantly associated with modern contraceptive use. These findings support the view that emotions and expectations are distinct and salient aspects of young women's fertility preferences. The expectations measure was not significantly related to modern contraceptive use, which demonstrates that all dimensions do not operate similarly across reproductive outcomes.

We explored new methods of measuring emotions and expectations with the TLT data. Existing survey questions on emotions often pertain to respondents' level of happiness with respect to previous pregnancies. We tested a prospective measure using information on respondents' feelings toward a potential pregnancy as good news or bad news (e.g., Sennott and Yeatman 2018). We also utilized a new measure of expectations about a future pregnancy that was based on responses to a creative exercise. This prospective approach helps avoid ex-post rationalization, a common issue with survey questions that ask women about pregnancies that occurred in the past (Günther and Harttgen 2016). Moreover, the predictive power of these new measures with respect to pregnancy and contraceptive use suggests that they should be employed in other surveys worldwide, where researchers can assess their universality or how they should be adapted by context.

Our study was subject to several limitations. First, the young age of the TLT sample limits the generalizability of our results to women of reproductive age in Malawi. Nevertheless, young women ages 15-25 are in the midst of transitions to marriage and parenthood. How they form and change various dimensions of fertility preferences is key to understanding reproductive decision-making during this life stage.

Second, our dependent variable, pregnancy status, had a relatively large percentage of missing information across survey waves, and we therefore imputed these missing data. As a robustness check, we replicated our analyses using the non-imputed sample and found substantially similar results (available on request), which increased our overall confidence in the findings.

A third limitation is that we were unable to fully explore the role of potentially ambiguous or uncertain responses provided by women who thought a future pregnancy would be neither good news nor bad news or who answered "don't know" regarding their emotions. The sample size was too small in these categories to enable us to carry out statistical analyses of their characteristics and behaviors. Ambivalence and uncertainty are important components of fertility decision-making (Aiken et al. 2016; Bernardi et al. 2015; Cutler 
et al. 2018) and are areas for continued research. Relatedly, we found that much of the literature on fertility preferences is atheoretical. One important avenue for future work is to formulate an overarching theoretical framework that encompasses multiple dimensions of fertility preferences and fully considers the role of ambivalence and uncertainty. ${ }^{5}$

Our study offers insights for enhancing reproductive health programs. The importance of the new prospective measures of emotions and expectations surrounding pregnancy points to the potential use of these measures to better assess individuals' motivations and tailor appropriate services for them (Aiken 2015; Lifflander et al. 2007; Speizer 2006). For example, the prospective measure of emotions could be used as a screening question to help distinguish between women who have more or less motivation to avoid pregnancy and who could thereby benefit from specific types of contraceptives. Furthermore, we uncovered apparent high unmet need among this population of young women. Further investigation into unmet need assessed by measures of emotions or expectations could help to uncover the sources of this need, such as lack of awareness, access, or both.

Ours was a first step in validating multiple measures of fertility preferences as salient in one population. Future research should move beyond reliance on single indicators of fertility preferences and the assumption that such measures account or proxy for other dimensions. This recommendation extends to Demographic and Health Surveys (DHS), which have been valuable sources of data on standard measures of fertility preferences for decades (for a review see Gipson et al. 2008). Next steps also include examining the intersection and incongruence across the three dimensions, or constructing a valid and reliable composite measure, both of which could more finely tune our understanding of the range of approaches women take to becoming pregnant and responding to pregnancy.

Acknowledgements This study uses data from Tsogolo la Thanzi, a research project designed by Jenny Trinitapoli and Sara Yeatman, and funded by Grants R01-HD058366 and R01-HD077873 from the National Institute of Child Health and Human Development (NICHD). The first author was supported by NICHD funding to the Population Research Institute at the Pennsylvania State University for Population Research Infrastructure (P2C HD041025) and Family Demography Training (T32 HD007514).

\footnotetext{
$\overline{5}$ See Aiken et al. (2016) for a conceptual framework aiming to inform women-centered approaches to helping them achieve their reproductive goals. The framework is not theoretically based but nevertheless considers the multidimensional nature of fertility preferences and reproductive behaviors.
}

\section{References}

Aiken, A. R. (2015). Happiness about unintended pregnancy and its relationship to contraceptive desires among a predominantly Latina cohort. Perspectives on Sexual and Reproductive Health, 47(2), 99-106.

Aiken, A. R., Borrero, S., Callegari, L. S., \& Dehlendorf, C. (2016). Rethinking the pregnancy planning paradigm: Unintended conceptions or unrepresentative concepts? Perspectives on Sexual and Reproductive Health, 48(3), 147.

Asare, M. (2015). Using the theory of planned behavior to determine the condom use behavior among college students. American Journal of Health Studies, 30(1), 43.

Barrett, G., \& Wellings, K. (2002). What is a 'planned' pregnancy? Empirical data from a British study. Social Science and Medicine, 55(4), 545-557.

Bernardi, L., Mynarska, M., \& Rossier, C. (2015). Uncertain, changing and situated fertility intentions. In D. Philipov, A. C. Liefbroer, \& J. E. Klobas (Eds.), Reproductive decision-making in a macromicro perspective (pp. 113-139). Dordrecht: Springer.

Casterline, J. B., \& Sinding, S. W. (2000). Unmet need for family planning in developing countries and implications for population policy. Population and Development Review, 26(4), 691-723.

Cutler, A., McNamara, B., Qasba, N., Kennedy, H. P., Lundsberg, L., \& Gariepy, A. (2018). "I Just Don't Know": An exploration of women's ambivalence about a new pregnancy. Women's Health Issues, 28(1), 75-81.

Dodoo, F. N. A., \& Tempenis, M. (2002). Gender, power, and reproduction: rural-urban differences in the relationship between fertility goals and contraceptive use in kenya. Rural Sociology, 67(1), 46-70.

Geist, C., Aiken, A. R., Sanders, J. N., Everett, B. G., Myers, K., Cason, P., et al. (2019). Beyond intent: Exploring the association of contraceptive choice with questions about Pregnancy Attitudes, Timing and How important is pregnancy prevention (PATH) questions. Contraception, 99(1), 22-26.

Gipson, J. D., Koenig, M. A., \& Hindin, M. J. (2008). The effects of unintended pregnancy on infant, child, and parental health: A review of the literature. Studies in Family Planning, 39(1), 18-38.

Gomez, A. M., Arteaga, S., Ingraham, N., Arcara, J., \& Villaseñor, E. (2018). It's not planned, but is it okay? The acceptability of unplanned pregnancy among young people. Women's Health Issues, 28(5), 408-414.

Günther, I., \& Harttgen, K. (2016). Desired fertility and number of children born across time and space. Demography, 53(1), 55-83.

Hartnett, C. S. (2012). Are Hispanic women happier about unintended births? Population Research and Policy Review, 31(5), 683-701.

Hayford, S. R. (2009). The evolution of fertility expectations over the life course. Demography, 46(4), 765-783.

Johnson, D. R., \& Young, R. (2011). Toward best practices in analyzing datasets with missing data: Comparisons and recommendations. Journal of Marriage and Family, 73(5), 926-945.

Jones, R. K. (2017). Change and consistency in US women's pregnancy attitudes and associations with contraceptive use. Contraception, 95(5), 485-490.

Klerman, L. V. (2000). The intendedness of pregnancy: A concept in transition. Maternal and Child Health Journal, 4(3), 155-162.

Lifflander, A., Gaydos, L. M., \& Hogue, C. J. R. (2007). Circumstances of pregnancy: Low income women in Georgia describe the difference between planned and unplanned pregnancies. Maternal and Child Health Journal, 11(1), 81-89.

Machiyama, K., Baschieri, A., Dube, A., Crampin, A. C., Glynn, J. R., French, N., et al. (2015). An assessment of childbearing preferences in northern Malawi. Studies in Family Planning, 46(2), 161-176. 
Morgan, S. P. (2001). Should Fertility Intentions Inform Fertility Forecasts? U.S. Census Bureau Conference: The Direction of Fertility in the United States.

Mumford, S. L., Sapra, K. J., King, R. B., Louis, J. F., \& Louis, G. M. B. (2016). Pregnancy intentions - a complex construct and call for new measures. Fertility and Sterility, 106(6), 1453-1462.

National Statistical Office (NSO) [Malawi], \& ICF Macro. (2017). Malawi demographic and health survey 2015-16. Rockville, MD: NSO and ORC Macro.

Rubin, D. B. (2004). Multiple imputation for nonresponse in surveys (Vol. 81). Hoboken: Wiley.

Rutstein, S. O., \& Johnson, K. (2004). DHS comparative reports 6 : The DHS wealth index. Measure DHS+. Calverton, Maryland: ORC Macro.

Ryder, N. B., \& Westoff, C. F. (1971). Reproduction in the United States, 1965. Princeton, New Jersey: Princeton University Press.

Santelli, J. S., Lindberg, L. D., Orr, M. G., Finer, L. B., \& Speizer, I. (2009). Toward a multidimensional measure of pregnancy intentions: Evidence from the United States. Studies in Family Planning, 40(2), 87-100.

Schoen, R., Astone, N. M., Kim, Y. J., Nathanson, C. A., \& Fields, J. M. (1999). Do fertility intentions affect fertility behavior? Journal of Marriage and the Family, 61, 790-799.

Sennott, C., \& Yeatman, S. (2018). Conceptualizing childbearing ambivalence: A social and dynamic perspective. Journal of Marriage and Family, 80(4), 888-901.
Speizer, I. S. (2006). Using strength of fertility motivations to identify family planning program strategies. International Family Planning Perspectives, 32, 185-191.

Stanford, J. B., Hobbs, R., Jameson, P., DeWitt, M. J., \& Fischer, R. C. (2000). Defining dimensions of pregnancy intendedness. Maternal and Child Health Journal, 4(3), 183-189.

Testa, M. R. (2012). Couple disagreement about short-term fertility desires in Austria: Effects on intentions and contraceptive behaviour. Demographic Research, 26, 63-98.

Testa, M. R., Cavalli, L., \& Rosina, A. (2011). Couples' childbearing behaviour in Italy: Which of the partners is leading it? Vienna Yearbook of Population Research, 9, 157-178.

Testa, M. R., \& Rampazzo, F. (2018). From intentions to births: Paths of realisation in a multi-dimensional life course. Vienna Yearbook of Population Research, 16, 1-22.

Trinitapoli, J., \& Yeatman, S. (2011). Uncertainty and fertility in a generalized AIDS epidemic. American Sociological Review, 76(6), 935-954.

Publisher's Note Springer Nature remains neutral with regard to jurisdictional claims in published maps and institutional affiliations. 